\section{The potential of mesenchymal stromal cells in immunotherapy}

\author{
"... a detailed understanding of the pathological processes (local \\ environment) accompanying different diseases is a prerequisite \\ before applying mesenchymal stromal cell therapy..."
}

First draft submitted: 9 March 2016; Accepted for publication: 14 April 2016; Published online: 6 July 2016

Keywords: immunomodulation $\bullet$ immunotherapy $\bullet$ mesenchymal stromal cells $\bullet$ MSCs - MSC derivative products

Over the past years, extensive studies have been conducted to develop safe and effective therapeutic strategies to treat human diseases. Immunomodulation refers to the manipulation of the immune system either by enhancing (immunopotentiation strategy), or supressing (immunosuppression strategy) the immune responses. Immunomodulation can be achieved by different agents, referred to as immunomodulators, including monoclonal antibodies, cytokines, glucocorticoids, adjuvants, ultraviolet light, plasmapheresis and many others [1]. Mesenchymal stromal cells (MSCs) are immunomodulators of special interest and have been suggested as promising therapeutics for several autoimmune diseases and transplantation complications [2]. According to the International Society for Cellular Therapy, MSCs are defined as plastic-adherent cells with a specific surface antigen expression profile $\left(\mathrm{CD}_{3}{ }^{+}, \mathrm{CD}^{+}{ }^{+}, \mathrm{CD}_{105}{ }^{+}, \mathrm{CD} 344^{-}, \mathrm{CD} 45^{-}\right.$, CD11b-, CD14-, CD19-, CD79a and HLA $\left.\mathrm{DR}^{-}\right)$and have the potential to differentiate, under standard in vitro differentiation conditions, into adipocytes, chondroblasts and osteoblasts [3]. Being a major component of the hematopoietic stem cell (HSC) niche, MSCs were first isolated from the bone marrow and identified for their capacities to support hematopoiesis [4], thus being of special importance for treating hematological disorders. Later, MSC-like cells were found and isolated from a variety of adult and fetal tissues including adipose tissue, umbilical cord and placenta [5]. This wide distribution in different parts of the organism highlighted a potential role for MSCs in tissue repair, and regeneration throughout individual's life.

The discovery of the superior immuneregulatory capacities of MSCs over different immune cells, raised their medical importance as they are used, nowadays, in preclinical and clinical studies as a potential therapy for treating autoimmune diseases, and modulating inflammatory responses [6]. The rationale of MSC-based therapy, is derived from their special immunologic profile and potent immunomodulatory capacity. Although MSCs isolated from different tissues exhibit differences in their expression profiles of some endothelial- and stromal-associated markers, all MSCs show low expression levels of different surface antigens, including MHC class I and the costimulatory molecules (CD40, CD80 and CD86) as well as no MHC class II expression [7]. Thanks to this special immunologic profile, MSCs can evade being rejected by host immune responses, thus contributing for their hypoimmunogenicity. Besides not being recognized as alloantigens, MSCs can actively sense the surrounding inflammatory microenvironment and modulate, accordingly, the function of different immune cells within the host [8]. In fact, different parameters including the concentration

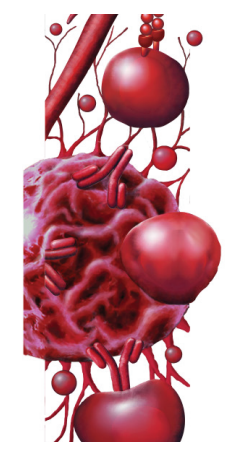

Mohammad Fayyad-Kazan ${ }^{\dagger, 1}$, Hussein Fayyad-Kazan, ${ }^{*, 2}$, 'Institut de Biologie et de Médecine Moléculaires, Université Libre de Bruxelles, 6041 Gosselies, Belgium 2Laboratory of Cancer Biology \& Molecular Immunology, Faculty of Sciences I, Lebanese University, Hadath, Lebanon

*Author for correspondence:

Tel.: +961 70764400

hfayyadk@gmail.com

${ }^{+}$Authors contributed equally to this work

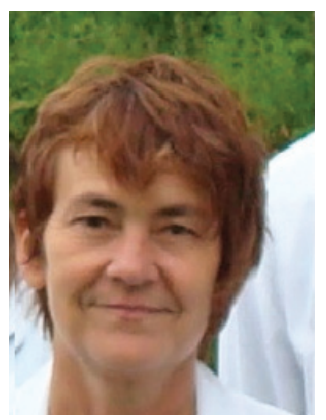

Laurence Lagneaux Laboratory of Clinical Cell Therapy, Université Libre de Bruxelles (ULB), Institut Jules Bordet, Brussels, Belgium

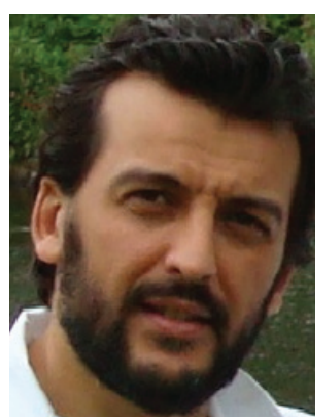

Mehdi Najar

Laboratory of Clinical Cell Therapy, Université Libre de Bruxelles (ULB), Institut Jules Bordet, Brussels, Belgium

Future $\because \%$ Medicine part of 
and type of cytokines present in the surrounding microenvironment can influence the immunologic phenotype and behavior of MSCs, demonstrating the plasticity of their immunomodulatory capacity [9]. When present in an inflammatory milieu, proinflammatory cytokines (such as TNF- $\alpha$ and INF- $\gamma$ ) will activate, via TLR3, MSCs which in turn adopt an immunosuppressive phenotype [8,9]. However, when inflammation is absent, activation of TLR4 renders MSCs in a proinflammatory phenotype [8,9]. The potent immunosuppressive and anti-inflammatory capacities of MSCs involves interaction with various innate and adaptive immune cells via different mechanisms. Paracrine signaling is the primarily mechanism for MSCs-dependent immunosuppression and involves different soluble molecules including TFG$\beta$, HGF, IL-10, SEMA-3A, PGE2, GAL, IDO, HO, TSG-6 [10,11]. Cell-to-cell direct contact is another mechanism for MSC-mediated immunosuppression that involves physical interaction between different MSC membrane-attached antigens such as CD200 ligand and ephrin (Eph) receptor with their respective targets, CD200 receptor (CD200R) and Eph ligand, expressed on immune cells [11].

Within the innate immune system, dendritic cells, serving as professional antigen-presenting cells (APCs), macrophages, monocytes and natural killer (NK) cells are major targets for MSC-mediated immunomodulation. In fact, MSCs can alter the generation of DCs by impairing their differentiation and maturation [12]. Theses immature DCs have tolerogenic features as they exhibited reduced ability to activate $T$ cells, secreted the anti-inflammatory cytokine IL-10 and inhibited the secretion of pro-inflammatory factors such as TNF- $\alpha$ and IL-12 [12]. According to their environmental signals, macrophages can adopt the M1 (pro-inflammatory) or M2 (antiinflammatory) phenotype. In the presence of MSCs, M1 macrophages are shifted into M2 cells, being characterized by a high production of IL-10 and low levels of TNF and INF- $\gamma$ [13]. When co-cultured with MSCs, NK cells, serving important role in allograft rejection since they can discriminate between self and non-self, became less activated with a reduced INF- $\gamma$ secretion and an impaired cytotoxic capacity [14].

In the adaptive immune system, MSCs are able to impair $\mathrm{T}$ cells activation, proliferation and differentiation [15]. During inflammatory conditions, MSCs can shift the differentiation of naive $\mathrm{CD}^{+}$from cells producing proinflammatory Th1 cytokines into cells secreting anti-inflammatory Th2 molecules [16]. Besides suppressing the differentiation of $\mathrm{CD}^{+}{ }^{+} \mathrm{Th} 0$ into proinflammatory Th17 cells, MSCs can stimulate the differentiation of Th0 cells into regulatory
T cells (Tregs) [17] that are important for conditioning immune responses. The effect of MSCs on B lymphocytes is contradictory and poorly understood. Some reports describe an inhibitory effect of MSCs on $\mathrm{B}$ cells proliferation, terminal differentiation and immunoglobulin production [18]. Currently, it is also reported that MSCs stimulate the differentiation of $\mathrm{B}$ cells into regulatory B cells (Bregs) that participate in ensuring tolerance upon suppressing the immune responses [19].

The in vitro and in vivo MSCs-immunosuppressive capacity inspired the development of MSC-based therapy. In vitro preclinical and clinical studies were performed to assess the MSCs-therapeutic efficacy in treating different immune-based diseases including allograft rejection, graft-versus-host disease (GVHD), myocardial inflammation, Crohn's disease, Multiple Sclerosis, Systemic Lupus Erythematosus and Rheumatoid Arthritis. The outcome was not totally satisfactory due to the critical discrepancy between expected and actual results. These discrepancies could be attributed to many causes where some important variables are currently highlighted in literature [20]. The procedures involving isolation, culture, expansion and cryopreservation of MSCs are all critical parameters. The age of the donor, the source of MSCs, the dose and frequency of injected cells as well as the transfusion method significantly impact the curative efficacy of MSCs. Nowadays, attention is focused towards the in vitro manipulation of MSCs. As described above, MSCsimmunosuppressive capacity is not constitutive but rather needs to be triggered either by potent inflammatory cytokines such as TNF- $\alpha$, INF- $\gamma$, IL- $\alpha$ and/ or IL-1 $\beta$ or by activating TLR3 signaling pathway. Hence, a detailed understanding of the pathological processes (local environment) accompanying different diseases is a prerequisite before applying MSC therapy. Besides, the timing of MSCs administration is critical since the inflammatory environment can change during the course of disease, thus the plasticity of MSCs can be affected. Cell therapy based on the use of MSCs and/or and their derivatives as an alternate source of biological products for therapeutic purposes, particularly for the management of immunological disorders, is gaining more and more notoriety. Nowadays, extensive studies are concerned with dissecting the molecular mechanisms by which different MSCs sense the surrounding inflammatory milieu and translate these messages into immunosuppressive responses. In parallel, much effort is dedicated for the exogenous and endogenous manipulation of MSCs in order to improve their immunotherapeutic efficacy. 
Currently, several challenges are being addressed and have to be well investigated:

- As MSCs are present in all tissues, can we specifically identify and mobilize in vivo a type of 'immunosuppressive MSCs' to avoid allogeneic transplantation?

- As MSCs harbor different immunoregulatory pathways, can we target a relevant active regulatory factor that will ensure their therapeutic effects?

- As MSCs-immunomodulatory capacities are enhanced by inflammatory priming, can we develop therapeutically relevant cells for clinical uses (with increased homing potential to damaged tissues and/or increased production of immunoregulatory molecules)?

- As MSCs could readily be genetically engineered, can they be used as cellular therapeutic vehicles to selectively deliver potent immunoregulatory factors?

- As soluble factors play critical roles in mediating MSCs-immunomodulatory effects, can those cells be replaced by their immunosuppressive supernatant?

- As MSCs can mediate, in part, their immunomodulatory effects through secreted exosomes

\section{References}

1 Dhama K, Saminathan M, Jacob SS et al. Effect of immunomodulation and immunomodulatory agents on health with some bioactive principles, modes of action and potent biomedical applications. Int. J. Pharmacol. 11(4), 253-290 (2015).

2 Sánchez-Berná I, Santiago-Díaz C, Jiménez-Alonso J. Immunomodulatory properties of stem mesenchymal cells in autoimmune diseases. Med. Clin. 144(2), 88-91 (2015).

3 Dominici M, Le Blanc K, Mueller I et al. Minimal criteria for defining multipotent mesenchymal stromal cells. The International Society for Cellular Therapy position statement. Cytotherapy 8(4), 315-317 (2006).

4 Friedenstein AJ, Chailakhjan RK, Lalykina KS. The development of fibroblast colonies in monolayer cultures of guinea-pig bone marrow and spleen cells. Cell Tissue Kinet. 3(4), 393-403 (1970).

5 Hoogduijn MJ, Betjes MGH, Baan CC. Mesenchymal stromal cells for organ transplantation: different sources and unique characteristics? Curr. Opin. Organ Transplant. 19(1), 41-46 (2014).

6 Kim N, Cho S-G. Clinical applications of mesenchymal stem cells. Korean J. Intern. Med. 28(4), 387-402 (2013).

7 Najar M, Raicevic G, Fayyad-Kazan H et al. Immunerelated antigens, surface molecules and regulatory factors in human-derived mesenchymal stromal cells: the expression carrying different bioactive molecules, could these microvesicles be selectively isolated and infused instead of the cells?

Due to their specific immunobiology, and in particular their immunomodulatory properties, the use of MSCs and/or and their derivatives in the field of immunotherapy is a major concern. As immunotherapeutic products, their use is becoming highly interesting and promising for the management of inflammatory and immunological disorders. However, before proceeding with clinical applications, the aims and challenges emerging from such field have to be addressed in order to improve the immunotherapeutic efficacy as well as ensure the safety of MSC-based immunotherapy.

\section{Financial \& competing interests disclosure}

Mohammad Fayyad-Kazan is awardee of a Televie postdoctoral fellowship. The authors have no other relevant affiliations or financial involvement with any organization or entity with a financial interest in or financial conflict with the subject matter or materials discussed in the manuscript apart from those disclosed.

No writing assistance was utilized in the production of this manuscript.

and impact of inflammatory priming. Stem Cell Rev. 8(4), 1188-1198 (2012).

8 Bernardo ME, Fibbe WE. Mesenchymal stromal cells: sensors and switchers of inflammation. Cell Stem Cell 13(14), 392-402 (2013).

9 Wang Y, Chen X, Cao W et al. Plasticity of mesenchymal stem cells in immunomodulation: pathological and therapeutic implications. Nat. Immunol. 15(11), 1009-1016 (2012).

10 Kim N, Cho S-G. New strategies for overcoming limitations of mesenchymal stem cell-based immune modulation. Int. J. Stem Cells 8(1), 54-68 (2015).

11 Najar M, Raicevic G, Crompot E et al. The immunomodulatory potential of mesenchymal stromal cells: a story of a regulatory network. J. Immunother. 39 (2), 45-59 (2016).

12 Spaggiari GM, Moretta L. Interactions between mesenchymal stem cells and dendritic cells. Adv. Biochem. Eng. Biotechnol. 130, 199-208 (2013).

13 Kim J, Hematti P. Mesenchymal stem cell-educated macrophages: a novel type of alternatively activated macrophages. Exp. Hematol. 37(12), 1445-1453 (2009).

14 Spaggiari GM, Capobianco A, Abdelrazik $\mathrm{H}$ et al. Mesenchymal stem cells inhibit natural killer-cell proliferation, cytotoxicity, and cytokine production: role of indoleamine 2,3-dioxygenase and prostaglandin E2. Blood 111(3), 1327-1333 (2008). 
15 Duffy MM, Ritter T, Ceredig R, Griffin MD. Mesenchymal stem cell effects on T-cell effector pathways. Stem Cell Res. Ther. 2(4), 34 (2011).

16 Aggarwal S, Pittenger MF. Human mesenchymal stem cells modulate allogeneic immune cell responses. Blood 1105(4), 1815-1822 (2005).

17 Ghannam S, Pène J, Moquet-Torcy G et al. Mesenchymal stem cells inhibit human Th17 cell differentiation and function and induce a $\mathrm{T}$ regulatory cell phenotype. $J$. Immunol. 185(1), 302-312 (2010).
18 Corcione A, Benvenuto F, Ferretti E et al. Human mesenchymal stem cells modulate B-cell functions. Blood 107(1), 367-372 (2006)

19 Franquesa M, Mensah FK, Huizinga R et al. Human adipose tissue-derived mesenchymal stem cells abrogate plasmablast formation and induce regulatory B cells independently of $\mathrm{T}$ helper cells. Stem Cells 33(3), 880-891 (2015).

20 Gao F, Chiu SM, Motan DAL et al. Mesenchymal stem cells and immunomodulation: current status and future prospects. Cell Death Dis. 7(1), e2062 (2016). 